\title{
FEATURE EXTRACTION FROM VRML MODELS FOR VIEW-BASED OBJECT RECOGNITION*
}

\author{
T. KRÜGER, J. WICKEL, P. ALVARADO, AND K.-F. KRAISS
}

Chair of Technical Computer Science, RWTH Aachen University, Germany

http://www.techinfo.rwth-aachen.de/

\begin{abstract}
View-based object recognition offers approaches to recognize three-dimensional objects from arbitrary two-dimensional views. In this paper we suggest different simple feature extraction methods based on three-dimensional object models. These are suited for both generating symbolic object descriptions and comparison with shape features extracted from images. We present a concept for improving view-based recognitions and provide first experimental results.
\end{abstract}

\section{Introduction}

Recognition of three-dimensional objects from arbitrary views is still an active field of research. There are several suggestions for matching an image (or an image sequence) of a presented object with a database containing information on three-dimensional objects. One way is the model-based recognition that tries to match a three-dimensional model with a model reconstructed from sample views. Some publications also suggest the selection and modification of a 3D model as search key for the query [4].

Another way is pursued by view-based search algorithms. This technique makes use of image processing to generate features (e. g. shape, edge, or texture descriptors), grouping and classification of the extracted features and matching with models stored in an object database [5]. This technique normally requires some kind of training process with a representative set of sample images.

View-based recognition offers two possible approaches for the compilation of the training database. The sample images can be acquired by taking representative pictures of the objects. If 3D data is available, the images may also be created by ray-tracing techniques [7]. The second approach offers the advantage that the 3D data can be used for further computations to support the recognition process.

Most features describing 3D objects that have been developed so far can only be used to compare 3D objects, but cannot be used for supporting a recognition based on images. For this reason, we present some features that are efficiently computable for $3 \mathrm{D}$ as well as $2 \mathrm{D}$ shapes.

* This work has been funded by the Heinz-Nixdorf Foundation. The test objects were kindly provided by fischerwerke Artur Fischer GmbH \& Co. KG. 


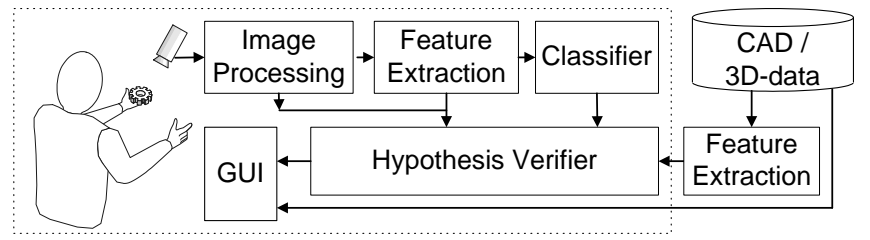

Figure 1. Architecture of a view-based recognition system using 3D information for verifying classifi cation results. A camera acquires a picture of an object which is processed and passed to a feature extraction module. The features are then handed to a classifi er, whose result may fi nally be verifi ed using independent data.

\section{Concept}

Object recognition systems usually calculate the best matching correspondence or sort objects by probability distributions. With an increasing size of the object set, this becomes more inaccurate and unreliable. Features extracted from 3D data provide additional information and enable the verification of hypotheses.

Figure 1 shows the architecture of the AXIOM system, which is our prototype implementation of the concept presented here [7]. A hypothesis verifier retrieves information from the image processing and feature extraction modules on the one hand and from the CAD database on the other hand. This verification can then be used to modify, reject, or confirm the classification result.

As an illustration of the way the validity is determined, consider the example of an aligned bounding box. A cubic object is always roughly projected to squares in camera images (see Fig. 2(a)). In contrast, lengthy objects can lead to different shapes in the two-dimensional projection as shown in Fig. 2(b).

(a)

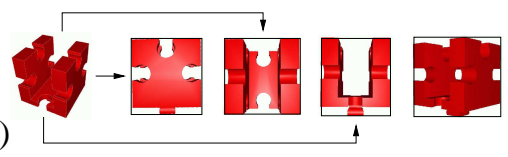

(b)

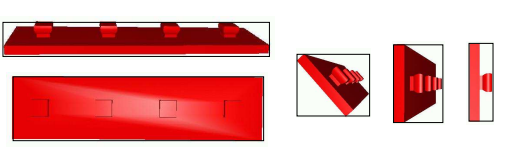

Figure 2. (a) Cubic object with different projections and the corresponding bounding boxes; (b) Different projections of a lengthy object and the corresponding bounding boxes.

When we compare the views shown in Fig. 2, it is obviously impossible for the left object (a) to generate a 2D bounding box similar to those shown on the left hand side of the right figure (b). Therefore, if such a view is to be recognized, we can safely regard the presence of the cubic object to be impossible.

\section{Shape features for three-dimensional object models}

One main goal of feature extraction methods is to yield features which are invariant against transformation and rotation. In the case of CAD information a third dimension has to be considered. The suggested methods in this section are 
intended for polygonal data. We use VRML files (Virtual Reality Modeling Language [1]) because of the availability of converters for different proprietary CAD formats.

\subsection{Basic features}

Various shape features for 3D objects have been proposed so far, ranging from simple geometric descriptions to statistical shape descriptions [3]. If we were to use these features in object recognition, we would have to reconstruct a 3D model from camera images. In order to avoid that process, we deploy selected simple features that can still be used to compare corresponding $2 \mathrm{D}$ features.

First, we developed an algorithm based on suggestions of [2], computing an oriented bounding box with minimal volume. As the concept is designed for an application operating with camera images, an absolute object length cannot be determined securely, but the quotient of the shortest to the medium and of the medium to the longest axis offer a representation that can be matched with bounding boxes extracted from two-dimensional images.

In addition to these measures, we developed an algorithm to approximate the global form of a regarded object (e.g. spheres, cylinders) based on the methods from [6] and [8]. Comparing the volumes of the surrounding shapes, the minimal and best fitting form is derived.

\subsection{Symbolic features}

Numerical object descriptions such as point sets are not as suitable for an effective user interaction as symbolic descriptions. It is therefore desirable to provide some kind of textual description of objects which can be derived from the the features described above: A comparison of the minimal surrounding shape volumes quickly yields the suitable global characterization (e.g. lengthy, flat), other discrete values can be converted by membership functions to textual features (e.g. complex, simple).

\section{Experimental Results}

For an assessment of the features described above, we tested them in a classification result verifier. This verifier receives the aligned bounding box of an object in a 2D-image. The feature used is the length of the longest axis of an object $c_{i}$ divided by the length of its shortest axis $\left(r_{3 \mathrm{D}}\left(c_{i}\right)\right)$. An analogous feature $\left(r_{2 \mathrm{D}}(x)\right)$ also exists for 2D bounding boxes of object images $x$. The validity $v_{i}$ of an object is 0 , if $r_{2 \mathrm{D}}(x)>s \cdot r_{3 \mathrm{D}}\left(c_{i}\right)$, and 1 otherwise. The scaling factor $s$ is used to allow for distortions caused by the projection of the object to the image plane. This leads to the distribution $P(C \mid V)=v_{i} / m$, with $m=\sum_{i} v_{i}$, which can be used to replace the prior $P(C)$ in maximum a-posteriori classification. This results in a new a-posteriori distribution for the classification result. The information gain of this refinement is $I G(C \mid V)=H(C)-H(C \mid V)$ with $H(C)$ denoting the entropy 
of the result distribution of the original classifier, and $H(C \mid V)$ the entropy of the refined distribution.

We performed an experiment with an uninformed classifier, which always recognizes a random object, so $P\left(C=c_{i}\right)=1 / n$ for an object set of $n$ objects. Obviously, this generates a distribution with entropy $H(C)=\log n$. The conditional entropy of the verified classification result is $H(C \mid V)=\frac{m}{n} \log m$. The object set for the experiment consisted of 50 objects (two are shown in Fig. 2); we used a scaling factor of $\sqrt{2}$.

The information gain was greater than zero for about $65 \%$ of the recognitions. The maximal information gain was 5 bits, which corresponds to $75 \%$ of the possible objects being rejected. When tested in combination with a "real" classifier trained on the same object set, using the described verifier increased the recognition rate from $45.2 \%$ to $48.3 \%$.

\section{Conclusion and Future Work}

In this paper we have presented a concept for refining results of visual object queries based on a combination of different algorithms to extract numerical and symbolic features from 3D models. The proposed features have the advantage that there is no need to reconstruct 3D models from camera images.

The experimental results so far are encouraging, but require further improvments. In the future, we plan to refine the verification process by including more sophisticated 3D features. We also aim to use the same principle described here to compare shapes of $3 \mathrm{D}$ and $2 \mathrm{D}$ objects.

\section{References}

1. R. Carey, G. Bell, and C. Marrin. Virtual Reality Modelling Language (VRML1997). ISO/IEC 14772-1, The VRML Consortium, 1997.

2. S. Gottschalk, M. C. Lin, and D. Manocha. OBBTree: A hierarchical structure for rapid interference detection. Computer Graphics, 30:171-180, 1996.

3. R. Osada, T. Funkhouser, B. Chazelle, and D. Dobkin. Matching 3D models with shape distributions. In Shape Modeling International, pages 154-166, Genova, Italy, 2001.

4. E. Paquet and M. Rioux. Nefertiti: a query by content system for three-dimensional model and image databases management. Image and Vision Computing, 17:157-166, 1999.

5. A. R. Pope. Model-based object recognition. a survey of recent research. Technical Report 94-04, Univ. of British Columbia, January 1994.

6. E. Welzl. Smallest enclosing disks (balls and ellipsoids). In H. Maurer, editor, New Results and New Trends in Computer Science, pages 359-370. Springer, 1991.

7. J. Wickel, P. Alvarado, P. Dörfler, T. Krüger, and K.-F. Kraiss. Axiom - a modular visual object retrieval system. In KI 2002: Advances in Artificial Intelligence, LNAI 2479, pages 253-267, Aachen, 2002.

8. Y. Zhou and S. Suri. Algorithms for minimum volume enclosing simplex in $R^{3}$. In Proceedings of the 11th Annual ACM-SIAM Symposium on Discrete Algorithms (SODA'00), pages 500-509, San Francisco, CA, 2000. 